\title{
Mordell-Lang conjecture for function fields in characteristic zero, revisited
}

\author{
Anand Pillay
}

\begin{abstract}
We give an elementary proof of the function field (or geometric) case of the Mordell-Lang conjecture in characteristic zero.
\end{abstract}

\section{Introduction}

In this paper a short proof of the following is given.

Theorem 1.1. Let $k<K$ be algebraically closed fields of characteristic zero. Let $A$ be a semiabelian variety over $K, X$ an irreducible subvariety of $A$ also over $K$, and $\Gamma$ a subgroup of $A(K)$ of finite rational rank (that is $\Gamma \otimes_{\mathbb{Z}} \mathbb{Q}$ is finite-dimensional as a $\mathbb{Q}$-vector space). Suppose that $\Gamma \cap X(K)$ is Zariski-dense in $X$ and that $X$ has trivial stabilizer in $A$. Then, after possibly replacing $X$ by a translate, there are a semi-abelian subvariety $A_{1}$ of $A$ containing $X$, and an isomorphism $f$ of $A_{1}$ with a semi-abelian variety $A_{2}$ defined over $k$, such that $f(X)$ is also defined over $k$.

The proof uses the differential-algebraic set-up as in [Bui92b] and [Hru96] but replaces Buium's analytic arguments and Hrushovski's appeal to the deep results on Zariski geometries [HZ96] and some model theory of groups of finite Morley rank, by considerations from [PZ01] involving linear differential equations on $k$-jets. Buium [Bui92b] essentially proves Theorem 1.1 in the case where $A$ is an abelian variety. The full statement of Theorem 1.1 is proved in [Hru96]. As stated in [PZ01] the main result (Theorem 2.5) of [PZ01] (which is stated there in model-theoretic language) can be plugged into the final part of Hrushovski's proof of Theorem 1.1 to give a substantial simplification. Nevertheless, it is considered worthwhile to give a direct account of the simplified proof, avoiding model-theoretic language.

It is worth remarking that a much stronger theorem than Theorem 1.1 is true (the absolute Mordell-Lang conjecture), proved in full generality by McQuillan [McQ95], using heavily work and ideas of Faltings [Fal91], Vojta [Voj96], Raynaud [Ray83], and Hindry [Hin88]. Namely under the hypotheses of Theorem 1.1, the conclusion is that $X$ is trivial (a point). As far as it is understood the most difficult case is when $A$ is defined over a number field and when $\Gamma$ is finitely generated. For $A$ an abelian variety this is treated in [Fal94] and in general ( $A$ semi-abelian) in [Voj96]. Specialization and other arguments are used to reduce to this case. A discussion of some of these specialization arguments appears in [Maz00]. Of course, Theorem 1.1 also provides a rather direct reduction to the number field case.

Let us also remark that included in Theorem 1.1 is Mordell's conjecture over function fields, which was proved in the 1960s by Manin [Man63], with proofs by Grauert [Gra65] and Parshin

Received 3 July 2002, accepted in final form 22 September 2003. 2000 Mathematics Subject Classification 11J89, 14G25, $13 \mathrm{~N} 10$.

Keywords: semi-abelian variety, function field, Mordell-Lang conjecture.

The author was partially supported by NSF grants and a Humboldt Foundation Research Award.

This journal is (C) Foundation Compositio Mathematica 2004. 
[Par68] following shortly thereafter. An account of the methods in these latter papers appears in Chapter VI of [Lan97].

The author considers it a major challenge to find an elementary proof of Theorem 1.1 in the positive characteristic case (and where prime to $p$ division points are used rather than all division points). The only known proof is Hrushovski's [Hru96] which makes use of the Zariski geometries results applied to separably closed fields. As pointed out in [PZ01] the methods of the present paper work in the positive characteristic case if $A$ is an ordinary abelian variety. But this case is already treated in [AV92].

The referee pointed out to us that the idea of performing descent to the constants using the theory of linear differential equations together with a 'Gauss map' from the variety in question to a certain Grassmanian, already appears in [Bui94], where Buium gives an algebraic proof of Mordell-Lang for function fields of characteristic zero in the 'non-hyperelliptic case'. In [Bui94], the Grassmanian in question was associated to a tangent space. In the present paper, we use a Grassmanian of subspaces of a suitable jet space, and the hypothesis of 'non-hyperellipticity' in [Bui94] can be removed.

\section{Algebraic $D$-groups and the proof of Theorem 1.1}

The main new aspect of our treatment is a rather direct proof of a (new, although implicit in [PZ01]) 'isotriviality' result for 'algebraic $D$-groups'. The category of algebraic $D$-groups was introduced by Buium in [Bui92a]. We give a quick description of the category and then state and prove our main lemma.

We work in characteristic zero. Let $F$ be a field equipped with a derivation $\partial$. Let $k$ be the field of constants of $F$ (set of elements killed by $\partial$ ). We will take $(F, \partial)$ to be differentially closed, that is, any finite system of differential polynomial equations and inequations over $F$ with a common solution in a differential field extension of $(F, \partial)$ already has a solution in $F$. (However, all the definitions make sense for arbitrary $(F, \partial)$.) Buium defines an algebraic $D$-variety to be an irreducible variety $X$ over $F$ equipped with a derivation $\partial_{X}$ on the structure sheaf of $X$ which extends $\partial$. Algebraic $D$-varieties form a category in the natural way. If $G$ is a connected algebraic group over $F$, and $\partial_{G}$ respects multiplication and inversion (in the obvious sense), he speaks of an algebraic $D$-group. $\partial_{X}$ as above is a kind of twisted vector field on $X$ and it will be useful to make this formalism more explicit. We describe briefly a functor $\tau$ defined on varieties over $F$, taking account of the derivation $\partial$. If the variety $X$ is locally defined by a system of polynomial equations $P_{1}\left(X_{1}, \ldots, X_{n}\right)=0, \ldots, P_{r}\left(X_{1}, \ldots, X_{n}\right)=0$ over $F$, then $\tau(X)$ will be locally defined by these equations together with

$$
\sum_{i=1, \ldots, n}\left(\frac{\partial P_{j}}{\partial X_{i}}\right)\left(X_{1}, \ldots, X_{n}\right) v_{i}+P_{j}^{\partial}\left(X_{1}, \ldots, X_{n}\right)=0
$$

for $j=1, \ldots, r$ where $P^{\partial}$ is obtained from $P$ by hitting the coefficients of $P$ with the derivation $\partial$. $\tau(X)$ is a variety over $F$, irreducible if $X$ is smooth, and comes with a canonical surjection to $X$. If $X$ happens to be defined over $k, \tau(X)$ is precisely $T(X)$, the Zariski tangent bundle of $X . \tau$ is a functor, so if $G$ is an algebraic group over $F$, then $\tau(G)$ is equipped with an algebraic group structure such that $\tau(G) \rightarrow G$ is a homomorphism. (So for $G$ defined over $k$, this is the usual algebraic group structure on $T(G)$.) See [Mar00] for more details on the functor $\tau$ applied to algebraic groups.

We define an algebraic $D$-group to be a pair $(G, s)$ where $G$ is a connected algebraic group over $F$ and $s: G \rightarrow \tau(G)$ is a section which is also a homomorphism of algebraic groups (over $F$ ). If $X$ is a subvariety of $G$ we say that $X$ is a $D$-subvariety of $(G, s)$ if $s \mid X: X \rightarrow \tau(X) \subseteq \tau(G)$. If $f$ : $G_{1} \rightarrow G_{2}$ is a homomorphism of algebraic groups then the functoriality of $\tau$ yields a homomorphism 


\section{A. Pillay}

$\tau(f): \tau\left(G_{1}\right) \rightarrow \tau\left(G_{2}\right)$ commuting with the canonical surjections to $G_{1}, G_{2}$. So a morphism between algebraic $D$-groups $\left(G_{1}, s_{1}\right)$ and $\left(G_{2}, s_{2}\right)$ is a rational homomorphism $f: G_{1} \rightarrow G_{2}$ such that $\tau(f) \cdot s_{1}=s_{2} \cdot f$. This agrees with Buium's definitions.

We will call the algebraic $D$-group $(G, s)$ split, or strongly isotrivial, if there is an algebraic group $G_{0}$ defined over $k$ such that $(G, s)$ is isomorphic to $\left(G_{0}, s_{0}\right)$ where $s_{0}: G_{0} \rightarrow T\left(G_{0}\right)$ is the 0-section.

We now give some final notation. Given an algebraic $D$-variety $(X, s),(X, s)^{\sharp}$ (or just $X^{\sharp}$ if $s$ is understood) denotes $\{x \in X(F): \partial(x)=s(x)\}$. This is a Zariski-dense subset of $X(F)$. If, moreover, $(G, s)$ is an algebraic $D$-group, then $G^{\sharp}$ is a subgroup of $G(F)$. ( $G^{\sharp}$ is a finite-dimensional differential algebraic group in the sense of Kolchin, and every such group arises this way.)

Our main result is as follows.

Theorem 2.1. Suppose that $(G, s)$ is an algebraic D-group, $X$ is a $D$-subvariety of $(G, s)$, and $\operatorname{Stab}(X)=\{g \in G: g \cdot X=X\}$ is trivial, namely equals $\{e\}$ where $e$ is the identity element of $G$. Assume also that $e \in X$ and $X$ generates $G$ in the sense that multiplication takes some $X^{ \pm 1} \times X^{ \pm 1} \times \cdots \times X^{ \pm 1}$ onto $G$. Then $(G, s)$ is strongly isotrivial.

Before giving the proof let us remark that it is related to (and even motivated by) Campana's proof [Cam80] of a result of Ueno: if $A$ is a complex torus and $X$ an analytic subvariety of $A$ with trivial stabilizer, then $X$ is an algebraic variety.

Proof of Theorem 2.1. By assumption $e \in X$. For $p \geqslant 1$ let $j_{p}(G)_{e}$, the $p$-jet of $G$ at $e$, be the dual space to $m / m^{p+1}$ where $m$ is the maximal ideal of the local ring of $G$ at $e . j_{p}(G)_{e}$ is a finite-dimensional $F$-vector space. Similarly, if $Y$ is a subvariety of $G$ passing through $e$, we obtain $j_{p}(Y)_{e}$ as a $F$-subspace of $j_{p}(G)_{e}$. If $Y$ is a member of an algebraic family of subvarieties, all passing through $e$, then $Y$ is determined (in this family) by $j_{p}(Y)_{e} \subseteq j_{p}(G)_{e}$ for sufficiently large $p$. $\operatorname{As~} \operatorname{Stab}(X)$ is trivial, for $t_{1}, t_{2} \in G, X t_{1}^{-1}=X t_{2}^{-1}$ if and only if $t_{1}=t_{2}$. For $t \in X, X t^{-1}$ contains $e$. Thus, for sufficiently large $p$, the map taking $t \in X$ to $j_{p}\left(X t^{-1}\right)_{e}$ gives a birational embedding $h$ of $X$ into $G r_{r}\left(j_{p}(G)_{e}\right)$, the variety of $r$-dimensional subspaces of $j_{p}(G)_{e}$ for suitable values of $r$. Let us fix such $p$. By virtue of $s$, the local ring of $G$ at $e$ is equipped with a derivation extending $\partial$, making it into a differential ring. As $e \in G^{\sharp}$, all powers of the maximal ideal $m$ of this local ring are differential ideals. It follows that $V=m / m^{p+1}$ is equipped with the structure of a $\partial$-module $\left(V, D_{V}\right)$ over $F$. The dual space $j_{p}(G)_{e}=V^{*}$ of $V$ is equipped with the dual connection $D_{V^{*}}$ : $\left(D_{V^{*}}(\lambda)\right)(v)=\partial(\lambda(v))-\lambda\left(D_{V}(v)\right)$. So $\left(V^{*}, D_{V^{*}}\right)$ is a $\partial$-module. As $F$ is differentially closed, the theory of linear differential equations gives us a fundamental system of solutions of the equation $D_{V^{*}}=0$ on $V^{*}$, that is a tuple $d=\left(d_{1}, \ldots, d_{n}\right)$ of elements of $V^{*}$ which is simultaneously an $F$-basis of $V^{*}$ and a $k$-basis of the solution space $\left(V^{*}\right)^{\partial}$ of $D_{V^{*}}=0$. Note that $\left(V^{*}\right)^{\partial}$ is precisely the set of $\lambda \in V^{*}$ such that $\lambda\left(D_{V}(v)\right)=\partial(\lambda(v))$ for all $v \in V$. In any case, the basis $d$ yields an identification of $V^{*}$ with $F^{n}$ and $\left(V^{*}\right)^{\partial}$ with $k^{n}$.

Now suppose that $t \in X^{\sharp}$. Then $X t^{-1}$ is a $D$-subvariety of $G$ and $e \in\left(X t^{-1}\right)^{\sharp}$. $\left(X t^{-1}, s \mid\left(X t^{-1}\right)\right)$ imposes on the local ring of $X t^{-1}$ at $e$ a differential ring structure which is clearly induced by that on the local ring of $G$ at $e$. As $e \in\left(X t^{-1}\right)^{\sharp}$ the maximal ideal $m_{t}$, say, of the local ring of $\left(X t^{-1}\right)$ at $e$ is again a differential ideal. Let $W_{t}=j_{p}\left(X t^{-1}\right)_{e}$. Then $W_{t}$ is a $\partial$-submodule of $\left(V^{*}, D_{V^{*}}\right)$. As above, the solution space $W_{t}^{\partial}$ of $D_{V^{*}}=0$ on $W_{t}$ is Zariski-dense in $W_{t}$. Identifying $W_{t}$ with an $r$-dimensional subspace of $F^{n}$, via the basis $d$, we see that the set of $k$-points of $W_{t}$ is Zariski-dense, whence $W_{t}$ is defined over $k$. Thus $W_{t}=j_{p}\left(X t^{-1}\right)_{e}$ is a $k$-rational point of $G_{r}\left(F^{n}\right)$. We have obtained a birational isomorphism $h$ of $X$ with a subvariety $Y$ of $G r_{r}\left(F^{n}\right)$ such that, for generic $t \in X^{\sharp}, h(t)$ is rational over $k$. Note that, as $X^{\sharp}$ is Zariski-dense in $X, Y$ is defined over $k$. It is now rather routine, using Weil's theorem, to find an algebraic group $G_{0}$ defined over $k$ satisfying the conclusion of Theorem 2.1. We will give some details. 


\section{MordeLL-LANG CONJECTURE FOR FUNCTION FIELDS}

Let $K$ be an algebraically closed subfield of $F$ of finite transcendence degree over which all the data $G, s, X, d$ are defined and such that $K$ is algebraically independent from $k$ over $k_{0}=K \cap k$. There is no harm in assuming $F$ to be a universal differential field (in the sense of Kolchin). We can find a generic point $t$ of $X$ over $K$ such that $t \in X^{\sharp}$. From the previous paragraph we find some tuple $c$ from $k$, such that $t$ and $c$ are birational over $K$, that is $K(t)=K(c)$. Write $c=f(t)$ where $f$ is a $K$-rational generically defined generically invertible function on $X$. Let $t_{1}, \ldots, t_{s}$, say, be generic independent (over $K$ ) elements of $X$ such that the product $g$ of the $t_{i}$ in $G$ is a generic point of $G$ over $K$, and such that all $t_{i} \in X^{\sharp}$. Note that $g \in G^{\sharp}$. Let $c_{i}=f\left(t_{i}\right)$. So $c_{i} \in k$ are independent over $k_{0}$, and we find $g^{\prime} \in k_{0}\left(c_{1}, \ldots, c_{s}\right)$ birational with $g$ over $K$. Write $g^{\prime}=f^{\prime}(g)$; again $f^{\prime}$ is $K$-rational and invertible. Let $g_{1}, g_{2}$ be chosen exactly like $g$, and independent over $K$. Let $g_{i}^{\prime}=f^{\prime}\left(g_{i}\right)$ for $i=1,2$. Let $g_{3}$ be the product of $g_{1}$ and $g_{2}$ in $G$. So $g_{3} \in G^{\sharp}$ and $f^{\prime}\left(g_{3}\right)=g_{3}^{\prime}$ is rational over $k$ too. $g_{1}^{\prime}, g_{2}^{\prime}, g_{3}^{\prime}$ are generic points of a variety $W$ say defined over $k_{0}$, and each is $k_{0}$-rational over the other two. By virtue of the construction, $W$ is equipped with a generic group law and so is birationally isomorphic over $k_{0}$ to an algebraic group $G_{0}$ defined over $k_{0}$ such that $g_{3}^{\prime}=g_{1}^{\prime} \cdot g_{2}^{\prime}$. Moreover, $f^{\prime}$ induces a $K$-rational isomorphism of $G$ with $G_{0}$. By construction $f^{\prime}$ takes $G^{\sharp}$ to $G_{0}(k)$. It follows that $f^{\prime}$ is also an isomorphism of algebraic $D$-groups between $(G, s)$ and $\left(G_{0}, s_{0}\right)$, where $s_{0}: G_{0} \rightarrow T\left(G_{0}\right)$ is the 0 -section.

We now complete the proof of Theorem 1.1. The global structure of the proof remains that of Hrushovski [Hru96], and uses Buium's construction [Bui92b] of a 'finite-dimensional differential algebraic' subgroup of $A$ containing $\Gamma$.

Proof of Theorem 1.1. We may assume $\Gamma$ to be Zariski-dense in $A$. By possibly enlarging $K$ we can choose a derivation $\partial$ on $K$ whose field of constants is precisely $k$ and such that $(K, \partial)$ is differentially closed. We may assume $(K, \partial)$ is universal in the sense of Kolchin. Buium ( $\S 2$ of [Bui92b]) produces a finite-dimensional differential algebraic subgroup of $A(F)$ containing $\Gamma$. Strictly speaking he does this assuming $A$ to be an abelian variety. In [Hru96] it is stated that this also goes through in the semi-abelian case. Alternatively, it follows from statement C5 in Appendix C of [Bui92a]. In any case, in the algebraic D-group formalism, Buium's construction amounts to the following.

There is a connected commutative algebraic $D$-group $(G, s)$ and a surjective homomorphism (of algebraic groups) $\pi: G \rightarrow A$ such that:

i) $L=\operatorname{ker}(\pi)$ is unipotent (and is thus the unipotent radical of $G$, as $A$ is semi-abelian);

ii) $\pi \mid G^{\sharp}$ is injective;

iii) $\Gamma \subset \pi\left(G^{\sharp}\right)$.

Let $X_{1}$ be the Zariski closure in $G$ of $\pi^{-1}(X) \cap G^{\sharp}$. Then $X_{1}$ is a (possibly reducible) $D$-subvariety of $G$. Replacing $X_{1}$ by a suitable irreducible component, we obtain a $D$-subvariety $X_{2}$ of $G$ such that $\pi$ maps $X_{2}$ into a Zariski-dense subset of $X$ (using the Zariski-denseness of $\Gamma \cap X$ in $X)$.

Claim. $X_{2}$ has trivial stabilizer in $G$.

Proof. $\operatorname{Stab}\left(X_{2}\right)$ is a $D$-subgroup of $G$, so if non-trivial it has a non-trivial point $a$ in $G^{\sharp}$. But then $\pi(a)$ is a non-trivial point in $\operatorname{Stab}(X)$ in $A$, contradiction.

Replacing $X_{2}$ by a translate by a point of $X_{2}^{\sharp}$ we may assume that $e \in X_{2}$. Let $G_{0}$ be the connected algebraic subgroup of $G$ generated by $X$. Then $G_{0}$ is a $D$-subgroup of $(G, s)$ and $X_{2}$ a $D$-subvariety. By Theorem 2.1, we may assume that $\left(G_{0}, s\right)$ is defined over $k$ and $s$ is the 0 -section. But then $G_{0}^{\sharp}$ is precisely $G_{0}(k)$. As (by item i) $L=\operatorname{ker}(\pi)$ is the unipotent radical 


\section{MoRdeLL-LANG CONJECTURE FOR FUNCTION FIELDS}

of $G, L_{1}=\operatorname{ker}\left(\pi \mid G_{0}\right)$ is the unipotent radical of $G_{0}$, so if non-trivial it has non-trivial points in $G_{0}(k)$. But this contradicts item ii. Thus $\pi \mid G_{0}$ is an isomorphism with an algebraic subgroup $G_{1}$ of $G$. Clearly $\pi\left(X_{2}\right)$ is a translate of $X$. Finally, as $X_{2}$ is a $D$-subvariety of $G_{0}$ and $s=0$ we see that $X_{2}$ is defined over $k$. This completes the proof of Theorem 1.1.

\section{REFERENCES}

AV92 D. Abramovich and J.-F. Voloch, Towards a proof of the Mordell-Lang conjecture in characteristic p, Int. Math. Res. Notices 5 (1992), 103-115.

Bui92a A. Buium, Differential algebraic groups of finite dimension, Lecture Notes in Mathematics, vol. 1506 (Springer, Berlin, 1992).

Bui92b A. Buium, Intersections in jet spaces and a conjecture of S. Lang, Ann. Math. 136 (1992), 557-567.

Bui94 A. Buium, Geometry of differential polynomial functions II: algebraic curves, Amer. J. Math. 116 (1994), 785-818.

Cam80 F. Campana, Algébricité et compacité dans l'espace des cycles d'un espace analytique complexe, Math. Ann. 251 (1980), 7-18.

Fal91 G. Faltings, Diophantine approximation on abelian varieties, Ann. Math. 133 (1991), 549-576.

Fal94 G. Faltings, The general case of S. Lang's conjecture, in Barsotti Symposium, Perspectives in Mathematics, vol. 15 (Academic Press, San Diego, 1994).

Gra65 H. Grauert, Mordells Vermutung über rationale Punkte auf algebraischen Kurven und Funktionenkörper, Publ. Math. Inst. Hautes Etudes Sci. 15 (1965), 131-149.

Hin88 M. Hindry, Autour d'une conjecture de S. Lang, Invent. Math. 94 (1988), 575-603.

Hru96 E. Hrushovski, The Mordell-Lang conjecture for function fields, J. Amer. Math. Soc. 9 (1996), 667-690.

HZ96 E. Hrushovski and B. Zilber, Zariski geometries, J. Amer. Math. Soc. 9 (1996), 1-56.

Lan97 S. Lang, Survey of Diophantine Geometry (Springer, Berlin, 1997).

Man63 Yu. Manin, Rational points on algebraic curves over function fields, Izv. Akad. Nauk SSSR Ser. Mat 27 (1963), 1395-1440.

Mar00 D. Marker, Manin kernels, in Connections between model theory and algebraic and analytic geometry, ed. A. Macintyre, Quaderni di matematica, vol. 6 (University of Naples, 2000).

Maz00 B. Mazur, Abelian varieties and the Mordell-Lang conjecture, in Model theory, algebra, and geometry, eds D. Haskell, A. Pillay and C. Steinhorn, MSRI publications, vol. 39 (Cambridge University Press, 2000).

McQ95 M. McQuillan, Division points on semi-abelian varieties, Invent. Math. 120 (1995), 143-159.

Par68 A. N. Parshin, Algebraic curves over function fields, Izv. Akad. Nauk SSSR Ser. Mat. 32 (1968) $1145-1170$.

PZ01 A. Pillay and M. Ziegler, Jet spaces of varieties over differential and difference fields, Selecta Math., to appear.

Ray83 M. Raynaud, Sous-variétés d'une variété abélienne et points de torsion, in Arithmetic and geometry I, eds M. Artin and J. Tate, Progress in Mathematics, vol. 35 (Birkhäuser, Boston, 1983), 327-352.

Voj96 P. Vojta, Integral points on subvarieties of semiabelian varieties I, Invent. Math. 126 (1996), $133-181$.

Anand Pillay pillay@math.uiuc.edu

Department of Mathematics, University of Illinois at Urbana-Champaign, 273 Altgeld Hall, MC-382, 1409 West Green Street, Urbana, IL 61801, USA 